\title{
Any $l$-state solutions of the Hulthén potential by the asymptotic iteration method
}

\author{
O. Bayrak†‡, G. Kocak $\ddagger$ and I. Boztosun $\ddagger$ \\ $\dagger$ Yozgat Faculty of Arts and Sciences, Department of Physics, \\ Erciyes University, Yozgat, Turkey and \\ $\ddagger$ Faculty of Arts and Sciences, Department of Physics, \\ Erciyes University, Kayseri, Turkey
}

(Dated: August 2, 2018)

\begin{abstract}
In this article, we present the analytical solution of the radial Schrödinger equation for the Hulthén potential within the framework of the asymptotic iteration method by using an approximation to the centrifugal potential for any $l$ states. We obtain the energy eigenvalues and the corresponding eigenfunctions for different screening parameters. The wave functions are physical and energy eigenvalues are in good agreement with the results obtained by other methods for different $\delta$ values. In order to demonstrate this, the results of the asymptotic iteration method are compared with the results of the supersymmetry, the numerical integration, the variational and the shifted $1 / N$ expansion methods.
\end{abstract}

PACS numbers: 03.65.Ge, 34.20.Cf, 34.20.Gj

Keywords: asymptotic iteration method, eigenvalues and eigenfunctions, Hulthén potential, quasi-analytical solution. 


\section{INTRODUCTION}

Over the last decades, the energy eigenvalues and corresponding eigenfunctions between interaction systems have raised a great deal of interest in relativistic quantum mechanics as well as in non-relativistic quantum mechanics. The exact solution of the wave equations (relativistic or non-relativistic) are very important since the wave function contains all the necessary information regarding the quantum system under consideration. Analytical methods such as the supersymmetry (SUSY) [1] and the Nikiforov-Uvarov method (NU) 2] have been used to solve the wave equations exactly or quasi-exactly for non zero angular momentum quantum number $(l \neq 0)$ by means of a given potential. The radial Schrödinger equation for the Hulthèn potential is solved exactly by using several techniques [6, 7, 8] for $l=0$. For the case $l \neq 0$, the effective Hulthén potential can not be solved exactly, but a number of methods have been used to find the bound state energy eigenvalues numerically 9] and quasi-analytically such as the variational 9], perturbation 10], shifted $1 / N$ expansion [11], NU [12] and SUSY [13] methods. The Hulthén potential [6] is one of the important short-range potentials in physics and it potential has been applied to a number of areas such as nuclear and particle physics, atomic physics, condensed matter and chemical physics (see 9] and the references therein). Therefore, it would be interesting and important to solve the non-relativistic radial Schrödinger equation for this potential for $l \neq 0$, since it has been extensively used to describe the bound and the continuum states of the interaction systems. Recently, an alternative method, called as the asymptotic iteration method (AIM), has been developed by Çiftçi et al. [3, 4] for solving second-order homogeneous linear differential equations and it has been applied to solve the non-relativistic radial Schrödinger equation or the relativistic Dirac equation.

In this paper, we aim to solve the Hulthén potential to obtain the energy eigenvalues and corresponding eigenfunctions for any $l$ states. In the next section, AIM is introduced. Then, in section III, the Schrödinger equation is solved by using AIM for the Hulthén potential for any $l$ states and our AIM results are given in comparison with the results of the numerical integration [9], the variational [9], the shifted $1 / N$ expansion [11] and the SUSY [13] methods. Finally, section IV] is devoted to the summary and conclusion. 


\section{OVERVIEW OF THE ASYMPTOTIC ITERATION METHOD}

\section{A. Energy Eigenvalues}

AIM is briefly outlined here and the details can be found in references [3, 4, 5]. AIM is proposed to solve the second-order differential equations of the form

$$
y^{\prime \prime}=\lambda_{0}(x) y^{\prime}+s_{0}(x) y
$$

where $\lambda_{0}(x) \neq 0$ and the prime denotes the derivative with respect to $x$. The variables, $s_{0}(x)$ and $\lambda_{0}(x)$, are sufficiently differentiable. The differential equation (11) has a general solution [3]

$$
y(x)=\exp \left(-\int^{x} \alpha\left(x_{1}\right) d x_{1}\right)\left[C_{2}+C_{1} \int^{x} \exp \left(\int^{x_{1}}\left[\lambda_{0}\left(x_{2}\right)+2 \alpha\left(x_{2}\right)\right] d x_{2}\right) d x_{1}\right]
$$

for sufficiently large $k, k>0$, if

$$
\frac{s_{k}(x)}{\lambda_{k}(x)}=\frac{s_{k-1}(x)}{\lambda_{k-1}(x)}=\alpha(x), \quad k=1,2,3, \ldots
$$

where

$$
\begin{aligned}
& \lambda_{k}(x)=\lambda_{k-1}^{\prime}(x)+s_{k-1}(x)+\lambda_{0}(x) \lambda_{k-1}(x) \\
& s_{k}(x)=s_{k-1}^{\prime}(x)+s_{0}(x) \lambda_{k-1}(x), \quad k=1,2,3, \ldots
\end{aligned}
$$

Note that one can also start the recurrence relations from $k=0$ with the initial conditions $\lambda_{-1}=1$ and $s_{-1}=0$ [20]. For a given potential such as the Hulthén, the radial Schrödinger equation is converted to the form of equation (11). Then, $\mathrm{s}_{0}(x)$ and $\lambda_{0}(x)$ are determined and $\mathrm{s}_{k}(x)$ and $\lambda_{k}(x)$ parameters are calculated by the recurrence relations given by equation (4).

The termination condition of the method in equation (3) can be arranged as

$$
\Delta_{k}(x)=\lambda_{k}(x) s_{k-1}(x)-\lambda_{k-1}(x) s_{k}(x)=0 \quad k=1,2,3, \ldots
$$

The energy eigenvalues are obtained from the roots of the equation (5) if the problem is exactly solvable. If not, for a specific $n$ principal quantum number, we choose a suitable $x_{0}$ point, determined generally as the maximum value of the asymptotic wave function or the minimum value of the potential [3, 18, 19, 20], and the approximate energy eigenvalues are obtained from the roots of this equation for sufficiently great values of $k$ with iteration. 


\section{B. Energy Eigenfunctions}

In this study, we seek the exact solution of the radial Schrödinger equation for which the relevant second order homogenous linear differential equation takes the following general form [4],

$$
y^{\prime \prime}=2\left(\frac{a x^{N+1}}{1-b x^{N+2}}-\frac{(t+1)}{x}\right) y^{\prime}-\frac{w_{k}^{t}(N) x^{N}}{1-b x^{N+2}} y, \quad 0<x<\infty
$$

If this equation is compared to equation (11), it entails the following expressions

$$
\lambda_{0}(x)=2\left(\frac{a x^{N+1}}{1-b x^{N+2}}-\frac{(t+1)}{x}\right), \quad s_{0}(x)=-\frac{w_{k}^{t}(N) x^{N}}{1-b x^{N+2}}
$$

$a$ and $b$ are constants and $w_{k}^{t}(N)$ can be determined from the condition (3) for $k=0,1,2,3, \ldots$ and $N=-1,0,1,2,3, \ldots$ as follows:

$$
\begin{aligned}
w_{k}^{t}(-1) & =k(2 a+2 b t+(k+1) b) \\
w_{k}^{t}(0) & =2 k(2 a+2 b t+(2 k+1) b) \\
w_{k}^{t}(1) & =3 k(2 a+2 b t+(3 k+1) b) \\
w_{k}^{t}(2) & =4 k(2 a+2 b t+(4 k+1) b) \\
w_{k}^{t}(3) & =5 k(2 a+2 b t+(5 k+1) b) \\
\ldots \text { etc } &
\end{aligned}
$$

Hence, these formule are easily generalized as,

$$
w_{k}^{t}(N)=b(N+2)^{2} k\left(k+\frac{(2 t+1) b+2 a}{(N+2) b}\right)
$$

The exact eigenfunctions can be derived from the following generator:

$$
y_{n}(x)=C_{2} \exp \left(-\int^{x} \frac{s_{k}\left(x^{\prime}\right)}{\lambda_{k}\left(x^{\prime}\right)} d x^{\prime}\right)
$$

where $k \geq n, n$ represents the radial quantum number and $k$ shows the iteration number. For exactly solvable potentials, the radial quantum number $n$ is equal to the iteration number $k$ and the eigenfunctions are obtained directly from equation (14). For nontrivial potentials that have no exact solutions, $k$ is always greater than $n$ in these numerical solutions and the approximate energy eigenvalues are obtained from the roots of equation (5) for sufficiently great values of $k$ with iteration. It should be pointed out that $\alpha(x)$ given by equation (3) is 
equal to zero for the ground state. Therefore, using equation (3) with (7) in equation (14), the eigenfunctions are obtained as follows,

$$
\begin{aligned}
y_{0}(x) & =C_{2} \\
y_{1}(x) & =-C_{2}(N+2) \sigma\left(1-\frac{b(\rho+1)}{\sigma} x^{N+2}\right) \\
y_{2}(x) & =C_{2}(N+2)^{2} \sigma(\sigma+1)\left(1-\frac{2 b(\rho+2)}{\sigma} x^{N+2}+\frac{b^{2}(\rho+2)(\rho+3)}{\sigma(\sigma+1)} x^{2(N+2)}\right) \\
y_{3}(x) & =-C_{2} \frac{\sigma(\sigma+1)(\sigma+2)}{(N+2)^{-3}} \\
& \times\left(1-\frac{3 b(\rho+3)}{\sigma} x^{N+2}+\frac{3 b^{2}(\rho+3)(\rho+4)}{\sigma(\sigma+1)} x^{2(N+2)}-\frac{b^{3}(\rho+3)(\rho+4)(\rho+5)}{\sigma(\sigma+1)(\sigma+2)} x^{3(N+2)}\right)
\end{aligned}
$$

.. etc

Finally, the following general formula for the exact solutions $y_{n}(x)$ is obtained as,

$$
y_{n}(x)=(-1)^{n} C_{2}(N+2)^{n}(\sigma)_{n}{ }_{2} F_{1}\left(-n, \rho+n ; \sigma ; b x^{N+2}\right)
$$

It is important to note that square integrable one in $L^{2}$ is this total wave function which is the asymptotic form of the wave function times $y_{n}(x)$ given by equation (14). Here, $(\sigma)_{n}=\frac{\Gamma(\sigma+n)}{\Gamma(\sigma)}, \quad \sigma=\frac{2 t+N+3}{N+2} \quad$ and $\quad \rho=\frac{(2 t+1) b+2 a}{(N+2) b}$. The $(\sigma)_{n}$ and the ${ }_{2} F_{1}$ are known as the Pochhammer symbol and the Gauss hypergeometric function, respectively.

\section{CALCULATION OF THE ENERGY EIGENVALUES AND EIGENFUNC- TIONS}

The motion of a particle with the mass $M$ in the spherically symmetric potential is described in the spherical coordinates by the following Schrödinger equation:

$\frac{-\hbar^{2}}{2 M}\left(\frac{\partial^{2}}{\partial r^{2}}+\frac{2}{r} \frac{\partial}{\partial r}+\frac{1}{r^{2}}\left[\frac{1}{\sin \theta} \frac{\partial}{\partial \theta}\left(\sin \theta \frac{\partial}{\partial \theta}\right)+\frac{1}{\sin ^{2} \theta} \frac{\partial^{2}}{\partial \phi^{2}}\right]+V(r)\right) \Psi_{n l m}(r, \theta, \phi)=E \Psi_{n l m}(r, \theta, \phi)$

Defining $\Psi_{n l m}(r, \theta, \phi)=R_{n l}(r) Y_{l m}(\theta, \phi)$, we obtain the radial part of the Schrödinger equation:

$$
\left(\frac{d^{2}}{d r^{2}}+\frac{2}{r} \frac{d}{d r}\right) R_{n l}(r)+\frac{2 M}{\hbar^{2}}\left[E-V(r)-\frac{l(l+1) \hbar^{2}}{2 M r^{2}}\right] R_{n l}(r)=0
$$


It is sometimes convenient to define $R_{n l}(r)$ and the effective potential as follows

$$
R_{n l}(r)=\frac{u_{n l}(r)}{r}, \quad V_{e f f}=V(r)+\frac{l(l+1) \hbar^{2}}{2 M r^{2}}
$$

Since

$$
\left(\frac{d^{2}}{d r^{2}}+\frac{2}{r} \frac{d}{d r}\right) \frac{u_{n l}(r)}{r}=\frac{1}{r} \frac{d^{2}}{d r^{2}} u_{n l}(r)
$$

The radial Schrödinger equation [8] given by equation (17) follows that

$$
\frac{d^{2} u_{n l}(r)}{d r^{2}}+\frac{2 M}{\hbar^{2}}\left[E-V_{e f f}\right] u_{n l}(r)=0
$$

The Hulthén potential [6] is given by

$$
V_{H}(r)=-Z e^{2} \delta \frac{e^{-\delta r}}{1-e^{-\delta r}}
$$

where $\mathrm{Z}$ and $\delta$ are respectively the atomic number and the screening parameter, determining the range for the Hulthén potential. The Hulthén potential behaves like the Coulomb potential near the origin $(r \longrightarrow 0)$, but in the asymptotic region $(r \gg 1)$, the Hulthén potential decreases exponentially, so its capacity for bound states is smaller than the Coulomb potential. However, for small values of the screening parameter $\delta$, the Hulthén potential becomes the Coulomb potential given by $V_{C}=-\frac{Z e^{2}}{r}$. The effective Hulthén potential is

$$
V_{e f f}(r)=V_{H}(r)+V_{l}=-Z e^{2} \delta \frac{e^{-\delta r}}{1-e^{-\delta r}}+\frac{l(l+1) \hbar^{2}}{2 M r^{2}}
$$

where $V_{l}=\frac{l(l+1) \hbar^{2}}{2 M r^{2}}$ is known as the centrifugal term. This effective potential can not be solved analytically for $l \neq 0$ because of the centrifugal term. Therefore, we must use an approximation for the centrifugal term similar to other authors [12, 13, 14, 15, 16, 17]. In this approximation, $\frac{1}{r^{2}}=\delta^{2} \frac{e^{-\delta r}}{\left(1-e^{-\delta r}\right)^{2}}$ is used for the centrifugal term. As shown in figure 11, this approximation is only valid for small $\delta r$ and it breaks down in the high-screening region. For small $\delta r, \widetilde{V}_{e f f}(r)$ is very well approximated to $V_{e f f}(r)$ and the Schrödinger equation for this approximate potential is solvable analytically. So, the effective potential becomes

$$
\widetilde{V}_{e f f}(r)=-Z e^{2} \delta \frac{e^{-\delta r}}{1-e^{-\delta r}}+\frac{l(l+1) \hbar^{2} \delta^{2}}{2 M} \frac{e^{-\delta r}}{\left(1-e^{-\delta r}\right)^{2}}
$$

Instead of solving the radial Schrödinger equation for the effective Hulthén potential $V_{\text {eff }}(r)$ given by equation (22), we now solve the radial Schrödinger equation for the new effective potential $\widetilde{V}_{e f f}(r)$ given by equation (23). Inserting this new effective potential into 
equation (20) and using the following ansatzs in order to make the differential equation more compact

$$
-\varepsilon^{2}=\frac{2 M E}{\hbar^{2} \delta^{2}}, \quad \beta^{2}=\frac{2 M Z e^{2}}{\hbar^{2} \delta}, \quad \delta r=x
$$

The radial Schrödinger equation takes the following form:

$$
\frac{d^{2} u_{n l}(x)}{d x^{2}}+\left[-\varepsilon^{2}+\beta^{2} \frac{e^{-x}}{\left(1-e^{-x}\right)}-l(l+1) \frac{e^{-x}}{\left(1-e^{-x}\right)^{2}}\right] u_{n l}(x)=0
$$

If we rewrite equation (25) by using a new variable of the form $z=e^{-x}$, we obtain

$$
\frac{d^{2} u_{n l}(z)}{d z^{2}}+\frac{1}{z} \frac{d u_{n l}(z)}{d z}+\left[-\frac{\varepsilon^{2}}{z^{2}}+\frac{\beta^{2}}{z(1-z)}-\frac{l(l+1)}{z(1-z)^{2}}\right] u_{n l}(z)=0
$$

In order to solve this equation with AIM, we should transform this equation to the form of equation (11). Therefore, the reasonable physical wave function we propose is as follows

$$
u_{n l}(z)=z^{\varepsilon}(1-z)^{l+1} f_{n l}(z)
$$

If we insert this wave function into equation (26), we have the second-order homogeneous linear differential equations as in the following form

$$
\frac{d^{2} f_{n l}(z)}{d z^{2}}=\left[\frac{(2 \varepsilon+2 l+3) z-(2 \varepsilon+1)}{z(1-z)}\right] \frac{d f_{n l}(z)}{d z}+\left[\frac{(2 \varepsilon+l+2) l+2 \varepsilon-\beta^{2}+1}{z(1-z)}\right] f_{n l}(z)
$$

which is now amenable to an AIM solution. By comparing this equation with equation (II), we can write the $\lambda_{0}(z)$ and $s_{0}(z)$ values and by means of equation (4), we may calculate $\lambda_{k}(z)$ and $s_{k}(z)$. This gives:

$$
\begin{aligned}
\lambda_{0}(z) & =\left(\frac{(2 \varepsilon+2 l+3) z-(2 \varepsilon+1)}{z(1-z)}\right) \\
s_{0}(z) & =\left(\frac{(2 \varepsilon+l+2) l+2 \varepsilon-\beta^{2}+1}{z(1-z)}\right) \\
\lambda_{1}(z) & =\frac{2+6 \varepsilon-7 z-2 l z-\beta^{2} z+12 z^{2} l-18 \varepsilon z-6 \varepsilon z l}{z^{2}(-1+z)^{2}} \\
& +\frac{12 \varepsilon z^{2}+11 z^{2}+4 \varepsilon^{2}+l^{2} z+\beta^{2} z^{2}+4 \varepsilon^{2} z^{2}-8 \varepsilon^{2} z+6 \varepsilon z^{2} l+3 l^{2} z^{2}}{z^{2}(-1+z)^{2}} \\
s_{1}(z) & =\frac{\left(2 l+2 \varepsilon-\beta^{2}+2 \varepsilon l+l^{2}+1\right)(-2+5 z+2 \varepsilon z+2 l z-2 \varepsilon)}{z^{2}(-1+z)^{2}} \\
\ldots \text { etc } &
\end{aligned}
$$


Combining these results with the quantization condition given by equation (5) yields

$$
\begin{gathered}
s_{0} \lambda_{1}-s_{1} \lambda_{0}=0 \quad \Rightarrow \quad \varepsilon_{0}=\frac{\beta^{2}-1-2 l-l^{2}}{2(l+1)}, \quad \text { for } \quad k=1 \\
s_{1} \lambda_{2}-s_{2} \lambda_{1}=0 \quad \Rightarrow \quad \varepsilon_{1}=\frac{\beta^{2}-4-4 l-l^{2}}{2(l+2)}, \quad \text { for } \quad k=2 \\
s_{2} \lambda_{3}-s_{3} \lambda_{2}=0 \quad \Rightarrow \quad \varepsilon_{2}=\frac{\beta^{2}-9-6 l-l^{2}}{2(l+3)}, \quad \text { for } \quad k=3 \\
\ldots \text { etc }
\end{gathered}
$$

When the above expressions are generalized, the eigenvalues turn out as

$$
\varepsilon_{n l}=\left(\frac{\beta^{2}-(n+l+1)^{2}}{2(n+l+1)}\right) \quad n, l=0,1,2,3, \ldots
$$

Using equation (24), we obtain the energy eigenvalues $\mathrm{E}_{n l}$,

$$
E_{n l}=-\frac{\hbar^{2}}{2 M}\left[\frac{M Z e^{2}}{\hbar^{2}(n+l+1)}-\frac{(n+l+1) \delta}{2}\right]^{2}
$$

In the atomic units $(\hbar=M=e=1)$ and for $\mathrm{Z}=1$, equation (32) turns out to be

$$
E_{n l}=-\frac{1}{2}\left[\frac{1}{(n+l+1)}-\frac{(n+l+1) \delta}{2}\right]^{2}
$$

In order to test the accuracy of equation (333), we calculate the energy eigenvalues for $Z=1$, any $n$ and $l$ quantum numbers and several values of the screening parameter. AIM results are compared with the results of the numerical integration [9], the variational [9], the shifted 1/N expansion [11] and the SUSY [13] methods in Table I and Table II] As it can be seen from the results presented in these tables, the AIM results are in good agrement with the results of the other methods for the small $\delta$ values. For large $\delta$ values, there are differences between our results and the results of others. This difference is due to the $\widetilde{V}_{\text {eff }}(r)$ potential, which we have used to approximate the $V_{\text {eff }}(r)$ potential. As it is seen from figure 1. for large $\delta r$ values, the discrepancy becomes apparent between our $\widetilde{V}_{e f f}(r)$ and the true $V_{e f f}(r)$ potentials. This gives rise to the differences for the eigenvalues presented in Tables 【 and 【at large $\delta$ values.

Now, as indicated in Section II] we can determine the corresponding wave functions by using equation (15). When we compare equation (6) and equation (28), we find $N=-1$, $b=1, a=l+1$, and $t=\frac{2 \varepsilon-1}{2}$. Therefore, we find $\rho=2(\varepsilon+l+1)$ and $\sigma=2 \varepsilon+1$. So, we can easily find the solution for $f_{n l}(z)$, for the energy eigenvalue equation (32) by using 
equation (15).

$$
f_{n l}(z)=(-1)^{n} \frac{\Gamma\left(2 \varepsilon_{n}+n+1\right)}{\Gamma\left(2 \varepsilon_{n}+1\right)}{ }_{2} F_{1}\left(-n, 2 \varepsilon_{n}+2 l+2+n ; 2 \varepsilon_{n}+1 ; z\right)
$$

Thus, we can write the total radial wave function as below,

$$
u_{n l}(z)=N z^{\varepsilon_{n}}(1-z)^{l+1} F_{1}\left(-n, 2\left(\varepsilon_{n}+l+1\right)+n ; 2 \varepsilon_{n}+1 ; z\right)
$$

where $N$ is the normalization constant.

\section{CONCLUSION}

We have shown an alternative method to obtain the energy eigenvalues and corresponding eigenfunctions of the Hulthén potential within the framework of the asymptotic iteration method for any $l$ states. We have calculated the energy eigenvalues for the Hulthén potential with $Z=1$ and several values of the screening parameter. The wave functions are physical and energy eigenvalues are in good agreement with the results obtained by other methods. In order to demonstrate this, AIM results have been compared with the results of the numerical integration [9], the variational [9], the shifted $1 / N$ expansion [11] and the SUSY [13] methods in Tables \and III. For small $\delta$ values, AIM results are in good agreement with the results of the other methods, but in the high screening region, the agreement is poor. The reason is simply that when the $\delta r$ increases in the high screening region, the agreement between $V_{e f f}(r)$ and $\widetilde{V}_{\text {eff }}(r)$ potentials decreases as shown in Figure 1. This problem could be solved by making a better approximation of the centrifugal term.

It should be pointed out that the asymptotic iteration method gives the eigenvalues directly by transforming the radial Schrödinger equation into a form of $y^{\prime \prime}=\lambda_{0}(r) y^{\prime}+s_{0}(r) y$. The wave functions are easily constructed by iterating the values of $s_{0}(r)$ and $\lambda_{0}(r)$. The asymptotic iteration method results in exact analytical solutions if there is and provides the closed-forms for the energy eigenvalues as well as the corresponding eigenfunctions. Where there is no such a solution, the energy eigenvalues are obtained by using an iterative approach [18, 19, 20]. As it is presented, AIM puts no constraint on the potential parameter values involved and it is easy to implement. The results are sufficiently accurate for practical purposes. 


\section{Acknowledgments}

This paper is an output of the project supported by the Scientific and Technical Research Council of Turkey (TÜBİTAK), under the project number TBAG-106T024 and Erciyes University (FBA-03-27, FBT-04-15, FBT-04-16). Authors would also like to thank Dr. Ayṣe Boztosun for the proofreading as well as Professor R. L. Hall and Dr. H. Çiftçi for useful comments on the manuscript.

\section{REFERENCES}

[1] F. Cooper, A. Khare and U. Sukhatme, Phys. Rep. 251 (1995) 267.

[2] A. F. Nikiforov and V. B. Uvarov, Special Functions of Mathematical Physics, Birkhäuser, Basel, (1988).

[3] H. Ciftci, R. L. Hall and N. Saad, J. Phys. A: Math. Gen. 36 (2003) 11807.

[4] H. Ciftci, R. L. Hall and N. Saad, J. Phys. A: Math. Gen. 38 (2005) 1147.

[5] O. Bayrak and I. Boztosun, J. Phys. A: Math. Gen. 39 (2006) 6955.

[6] L. Hulthén, Ark. Mat. Astron. Fys. 28A (1942) 5.

[7] M. Akdas, R. Sever, J. Molec. Struc. (Theochem) 710 (2004) 223.

[8] S. Flügge, Practical Quantum Mechanics Vol. I, Springer, Berlin, (1994).

[9] Y.P. Varshni, Phys. Rev. A 4119904682

[10] P. Matthys, H. De Meyer, Phys. Rev. A 38 (1988) 1168.

[11] A.Z. Tang, F.T. Chan, Phys. Rev. A 35 (1987) 911.

[12] S. M. Ikhdair and R. Sever, arXiv:quant-ph/0508009.

[13] B. Gonul, O. Ozer, Y. Cancelik and M. Kocak, Phys. Lett. A 275 (2000) 238.

[14] R.L. Greene, C. Aldrich, Phys. Rev. A 14 (1976) 2363.

[15] U. Myhrman, J. Phys. A: Math. Gen. 16 (1983) 263.

[16] A. Bechlert, W Bühring, J. Phys. B: At. Mol. Opt. Phys. 21 (1988) 817.

[17] Shang-Wu Qian, Bo-Wen Huang, and Zhi-Yu Gu, New Journal of Physics 4 (2002) 13.

[18] O. Bayrak and I. Boztosun, Int. J. Quantum Chem. (2006), in press.

I. Boztosun, M. Karakoc, F. Yasuk and A. Durmus, J. Math. Phys. 47 (2006) 062301. 
[19] T. Barakat, J. Phys. A: Math. Gen. 39 (2006) 823.

T. Barakat, Phys. Lett. A344 (2005) 411.

T. Barakat, K. Abodayeh, A. Mukheimer, J. Phys. A: Math. Gen. 38 (2005) 1299.

[20] F. M. Fernández, J. Phys. A: Math. Gen. 37 (2004) 6173. 


\begin{tabular}{|c|c|c|c|c|c|c|}
\hline State & $\delta$ & AIM & SUSY [13] & Numerical Int. [9] & Variational [9] & Shifted $\frac{1}{N}$ [11] \\
\hline \multirow[t]{9}{*}{$2 \mathrm{p}$} & 0.025 & 0.1128125 & 0.1127605 & 0.1127605 & 0.1127605 & \\
\hline & 0.050 & 0.1012500 & 0.1010425 & 0.1010425 & 0.1010425 & 0.1010424 \\
\hline & 0.075 & 0.0903125 & 0.0898478 & 0.0898478 & 0.0898478 & \\
\hline & 0.100 & 0.0800000 & 0.0791794 & 0.0791794 & 0.0791794 & 0.0791794 \\
\hline & 0.150 & 0.0612500 & 0.0594415 & 0.0594415 & 0.0594415 & \\
\hline & 0.200 & 0.0450000 & 0.0418854 & 0.0418860 & 0.0418860 & 0.0418857 \\
\hline & 0.250 & 0.0312500 & 0.0266060 & 0.0266111 & 0.0266108 & \\
\hline & 0.300 & 0.0200000 & 0.0137596 & 0.0137900 & 0.0137878 & \\
\hline & 0.350 & 0.0112500 & 0.0036146 & 0.0037931 & 0.0037734 & \\
\hline \multirow[t]{5}{*}{$3 p$} & 0.025 & 0.0437590 & 0.0437068 & 0.0437069 & 0.0437069 & \\
\hline & 0.050 & 0.0333681 & 0.0331632 & 0.0331645 & 0.0331645 & 0.03316518 \\
\hline & 0.075 & 0.0243837 & 0.0239331 & 0.0239397 & 0.0239397 & \\
\hline & 0.100 & 0.0168056 & 0.0160326 & 0.0160537 & 0.0160537 & 0.01606772 \\
\hline & 0.150 & 0.0058681 & 0.0043599 & 0.0044663 & 0.0044660 & \\
\hline \multirow[t]{5}{*}{$3 \mathrm{~d}$} & 0.025 & 0.0437587 & 0.0436030 & 0.0436030 & 0.0436030 & \\
\hline & 0.050 & 0.0333681 & 0.0327532 & 0.0327532 & 0.0327532 & 0.0327532 \\
\hline & 0.075 & 0.0243837 & 0.0230306 & 0.0230307 & 0.0230307 & \\
\hline & 0.100 & 0.0168055 & 0.0144832 & 0.0144842 & 0.0144842 & 0.0144842 \\
\hline & 0.150 & 0.0058681 & 0.0132820 & 0.0013966 & 0.0013894 & \\
\hline
\end{tabular}

TABLE I: Energy eigenvalues of the Hulthén potential as a function of the screening parameter for $2 \mathrm{p}, 3 \mathrm{p}$ and $3 \mathrm{~d}$ states in atomic units $(\hbar=m=e=1)$ and for $Z=1$. 


\begin{tabular}{|c|c|c|c|c|c|c|}
\hline State & $\delta$ & AIM & SUSY [13] & Numerical Int. [9] & Variational [9] & Shifted $\frac{1}{N}$ [11] \\
\hline \multirow[t]{4}{*}{$4 \mathrm{p}$} & 0.025 & 0.0200000 & 0.0199480 & 0.0199489 & 0.0199489 & \\
\hline & 0.050 & 0.0112500 & 0.0110430 & 0.0110582 & 0.0110582 & 0.0110725 \\
\hline & 0.075 & 0.0050000 & 0.0045385 & 0.0046219 & 0.0046219 & \\
\hline & 0.100 & 0.0012500 & 0.0004434 & 0.0007550 & 0.0007532 & \\
\hline \multirow[t]{3}{*}{$4 \mathrm{~d}$} & 0.025 & 0.0200000 & 0.0198460 & 0.0198462 & 0.0198462 & \\
\hline & 0.050 & 0.0112500 & 0.0106609 & 0.0106674 & 0.0106674 & 0.0106690 \\
\hline & 0.075 & 0.0050000 & 0.0037916 & 0.0038345 & 0.0038344 & \\
\hline \multirow[t]{3}{*}{$4 \mathrm{f}$} & 0.025 & 0.0200000 & 0.0196911 & 0.0196911 & 0.0196911 & \\
\hline & 0.050 & 0.0112500 & 0.0100618 & 0.0100620 & 0.0100620 & 0.0100620 \\
\hline & 0.075 & 0.0050000 & 0.0025468 & 0.0025563 & 0.0025557 & \\
\hline \multirow[t]{2}{*}{$5 p$} & 0.025 & 0.0094531 & 0.0094011 & 0.0094036 & & 0.0094087 \\
\hline & 0.050 & 0.0028125 & 0.0026056 & 0.0026490 & & \\
\hline \multirow[t]{2}{*}{$5 \mathrm{~d}$} & 0.025 & 0.0094531 & 0.0092977 & 0.0093037 & & 0.0093050 \\
\hline & 0.050 & 0.0028125 & 0.0022044 & 0.0023131 & & \\
\hline \multirow[t]{2}{*}{$5 f$} & 0.025 & 0.0094531 & 0.0091507 & 0.0091521 & & 0.0091523 \\
\hline & 0.050 & 0.0028125 & 0.0017421 & 0.0017835 & & \\
\hline \multirow[t]{2}{*}{$5 g$} & 0.025 & 0.0094531 & 0.0089465 & 0.0089465 & & 0.0089465 \\
\hline & 0.050 & 0.0028125 & 0.0010664 & 0.0010159 & & \\
\hline $6 \mathrm{p}$ & 0.025 & 0.0042014 & 0.0041493 & 0.0041548 & & \\
\hline $6 \mathrm{~d}$ & 0.025 & 0.0042014 & 0.0040452 & 0.0040606 & & \\
\hline $6 f$ & 0.025 & 0.0042014 & 0.0038901 & 0.0039168 & & \\
\hline $6 \mathrm{~g}$ & 0.025 & 0.0042014 & 0.0036943 & 0.0037201 & & \\
\hline
\end{tabular}

TABLE II: Energy eigenvalues of the Hulthén potential as a function of the screening parameter for $4 \mathrm{p}, 4 \mathrm{~d}, 4 \mathrm{f}, 5 \mathrm{p}, 5 \mathrm{~d}, 5 \mathrm{f}, 5 \mathrm{~g}, 6 \mathrm{p}, 6 \mathrm{~d}, 6 \mathrm{f}$ and $6 \mathrm{~g}$ states in atomic units $(\hbar=m=e=1)$ and for $Z=1$. 


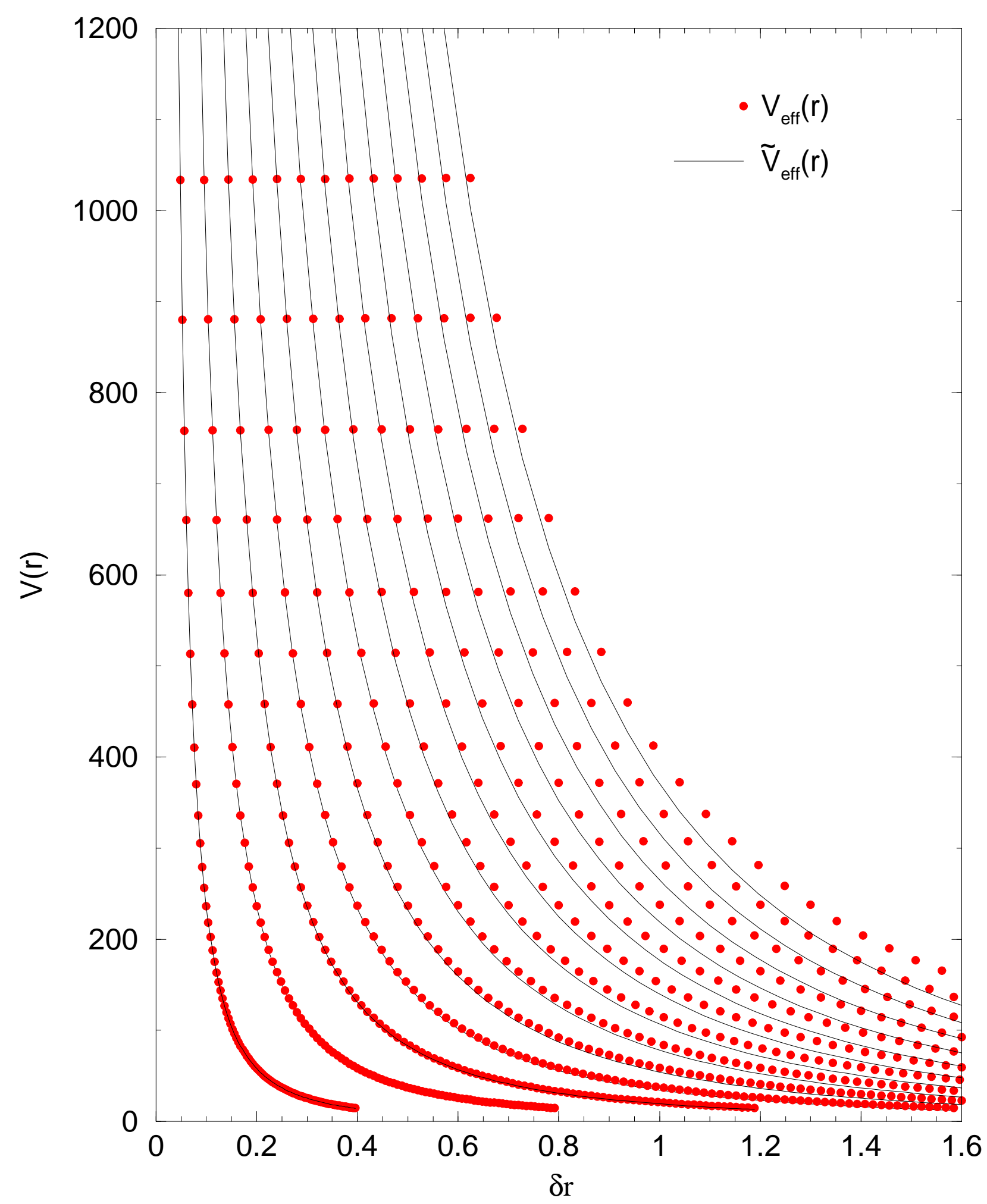

FIG. 1: The variation of the effective Hulthén $V_{\text {eff }}(r)$ and the approximate effective Hulthén potentials $\widetilde{V}_{\text {eff }}(r)$ with respect to the $\delta r$ for various values of the screening parameter. The parameters are in atomic units $(\hbar=e=m=1)$ and $\delta$ change from 0.4 to 5.2 in steps of 0.4 . 